\title{
Electrochemotherapy by pulsed electromagnetic field treatment (PEMF) in mouse melanoma B16F10 in vivo
}

\author{
Simona Kranjc도 Matej Kranjc², Janez Scancar ${ }^{3}$, Jure Jelenc ${ }^{4}$, Gregor Sersa ${ }^{1}$, \\ Damijan Miklavcic ${ }^{2}$
}

\author{
${ }^{1}$ Department of Experimental Oncology, Institute of Oncology Ljubljana, Ljubljana, Slovenia \\ 2 University of Ljubljana, Faculty of Electrical Engineering \\ 3 Jozef Stefan Institute, Ljubljana, Slovenia \\ ${ }^{4}$ Iskra Medical LLC, Ljubljana, Slovenia \\ Radiol Oncol 2016; 50(1): 39-48.
}

Received 30 October 2015

Accepted 20 January 2016

Correspondence to: Prof. Damijan Miklavčič, Ph.D., University of Ljubljana, Faculty of Electrical Engineering, Tržaška 25, SI-1000 Ljubljana, Slovenia. E-mail: damijan.miklavcic@fe.uni-lj.si

Disclosure: DM holds a patent on electrochemotherapy that have been licensed to IGEA S.p.a. and is also consultant to various companies having commercial interests in electroporation based treatments and therapies. Other co-authors have nothing to disclose.

Introduction. Pulsed electromagnetic field (PEMF) induces pulsed electric field, which presumably increases membrane permeabilization of the exposed cells, similar to the conventional electroporation. Thus, contactless PEMF could represent a promising approach for drug delivery.

Materials and methods. Noninvasive electroporation was performed by magnetic field pulse generator connected to an applicator consisting of round coil. Subcutaneous mouse B16F10 melanoma tumors were treated with intravenously injection of cisplatin (CDDP) $(4 \mathrm{mg} / \mathrm{kg}$ ), PEMF (480 bipolar pulses, at frequency of $80 \mathrm{~Hz}$, pulse duration of $340 \mu \mathrm{s}$ ) or with the combination of both therapies (electrochemotherapy - PEMF + CDDP). Antitumor effectiveness of treatments was evaluated by tumor growth delay assay. In addition, the platinum (Pt) uptake in tumors and serum, as well as Pt bound to the DNA in the cells and Pt in the extracellular fraction were measured by inductively coupled plasma mass spectrometry.

Results. The antitumor effectiveness of electrochemotherapy with CDDP mediated by PEMF was comparable to the conventional electrochemotherapy with CDDP, with the induction of 2.3 days and 3.0 days tumor growth delay, respectively. The exposure of tumors to PEMF only, had no effect on tumor growth, as well as the injection of CDDP only. The antitumor effect in combined treatment was related to increased drug uptake into the electroporated tumor cells, demonstrated by increased amount of Pt bound to the DNA. Approximately 2-fold increase in cellular uptake of Pt was measured.

Conclusions. The obtained results in mouse melanoma model in vivo demonstrate the possible use of PEMF induced electroporation for biomedical applications, such as electrochemotherapy. The main advantages of electroporation mediated by PEMF are contactless and painless application, as well as effective electroporation compared to conventional electroporation.

Key words: pulsed electromagnetic field; bipolar pulses; contactless electroporation; CDDP; electrochemotherapy; platinum determination; mouse melanoma

\section{Introduction}

Electroporation is a physical method enabling delivery of impermeable drugs, macromolecules, proteins and genetic material (plasmid DNA, siRNA, miRNA) into cells. ${ }^{1}$ Electroporation is related to the induced transmembrane voltage which if sufficiently high, enables the formation of tempo- 
rary structural changes in the plasma membrane and increases its permeability for molecules otherwise deprived of transmembrane transport mechanisms..$^{2-5}$ Electroporation of cells is predominantly induced by pulsed electric fields, which are generated with the train of square wave electric pulses of sufficient amplitude establishing local electric field (hundreds of $\mathrm{V} / \mathrm{cm}$ ). ${ }^{1,3,6,7}$ The electric field intensity and duration of the pulses determine whether the structural changes in the plasma membrane are reversible, allowing cells to survive, or irreversible, leading to cell death, due to the loss of homeostasis..$^{8-11}$ Nowadays, reversible electroporation is used as a platform technology ${ }^{12}$ and among others for drug delivery to various tissues, with therapeutic purposes for the treatment of cancer, known as electrochemotherapy. ${ }^{7,13-18}$

Electrochemotherapy is used in treatment of human cutaneous tumors of different histology, and has been translated also in treatment of deep seated tumors. ${ }^{15,18-22}$ In parallel, electrochemotherapy is being used for treatment of tumors in veterinary oncology..$^{23-26}$ The main chemotherapeutics used in electrochemotherapy are nonpermeable bleomycin and poorly permeable cisplatin (CDDP), via systemic or intratumoral administration route. Electric pulses can be delivered to the tumors via noninvasive plate electrodes, which embrace the tissue, or invasive needle electrodes, which are inserted into the tumor. ${ }^{7,27}$

In the past the effects of externally applied pulsed electromagnetic fields (PEMF) on the cells were studied extensively. It was demonstrated that externally applied PEMF can influence intracellular signal transduction, affect the cytoskeletal proteins involved in cell shape modification, induce changes in mitochondrial membrane potential, and besides that increase transmembrane molecular transport (electroporation). ${ }^{28-34}$ Since then, a few studies actually defined the PEMF parameters that enabled successful electropermeabilization of cells; i.e. large number of 25 up to 800 the $\mu$ s long magnetic field pulses applied at frequencies from $25 \mathrm{~Hz}$ up to $40 \mathrm{~Hz}$ and strength from $725 \mathrm{~V} / \mathrm{m}$ up to $160 \mathrm{kV} / \mathrm{m}^{30,35,36}$ Furthermore, its use as electroporation tool was shown in an approach for drug as well as for plasmid DNA delivery. ${ }^{36,37}$ Thus, such electromagnetic induction with alternating currents has the potential for simple contactless tissue electroporation, used for electrochemotherapy and gene electrotransfer.

The majority of studies with PEMF induced electroporation were using bipolar pulses. Generally, shorter and larger number of pulses resulted in better membrane permeabilization. ${ }^{30,34}$ In a recent study, time varying magnetic field of $6.1 \mathrm{~T}$ was shown as an interesting tool in drug delivery for antifungal treatment, as well as for irreversible electroporation. ${ }^{36,38}$ Furthermore, the bipolar pulses generated by magnetic field $(4 \mathrm{~T})$ were used for gene electrotransfer of plasmid DNA into the skin. ${ }^{37}$ Thus, as simple, noninvasive and contactless application of PEMF, which could enable electroporation of cells in the tissue, this approach showed the potential use for the clinical applications.

Due to only few studies in the field of electroporation induced by pulsed electromagnetic field we designed experiments and considered the use of such physical delivery technique in treatment of cancer, as a model for electroporation of tissues in vivo induced by PEMF. If feasible and effective, electroporation induced by PEMF would have the advantage over "conventional" electroporation, since it is noninvasive, contactless and does not induce pain during electroporation. We assessed the feasibility and antitumor effectiveness of electroporation induced with PEMF as drug delivery system for CDDP to murine melanoma B16F10 subcutaneous tumors. To prove the underlying mechanism of electroporation we measured the platinum $(\mathrm{Pt})$ bound to DNA in tumors. Electroporation induced by PEMF proved to facilitate drug uptake in tumors, such as CDDP, thus providing evidence of its feasibility and effectiveness.

\section{Materials and methods}

\section{Drug}

CDDP, a chemotherapeutic drug used in electrochemotherapy protocol in human and veterinary clinic, was chosen in the study to test the application of induced electroporation mediated with magnetic field. The stock solution of the chemotherapeutic drug used in the study, CDDP $(5 \mathrm{mg} / \mathrm{mL}$, Cysplatyl, Aventis Laboratory, Paris, France) was dissolved in aqua pro injection and frozen in aliquots of $1 \mathrm{~mL}$. In order that each animal received a dose of $80 \mu \mathrm{g}$ of CDDP, a fresh solution at appropriate concentration of CDDP $(1 \mathrm{mg} / \mathrm{mL})$ was prepared in $0.9 \%$ sodium chloride solution daily before each experiment.

\section{Mouse tumor model}

Female C57Bl/6 mice were purchased from Charles River Laboratories Italy s.r.l. (Calco, Italy) and were maintained in an adaptation period for 14 
days. They were kept at a constant room temperature with a 12 hours light cycle in a conventional animal facility. Eight-week old animals weighing 20-22 g were used in the experiments. Tumors in C57Bl/6 mice were implanted subcutaneously in the right flank of the mice by inoculation of suspension $1 \times 10^{6} \mathrm{~B} 16 \mathrm{~F} 10$ melanoma cells prepared in $100 \mu \mathrm{L}$ of phosphate-buffered saline (PBS) for electrochemotherapy experiments. All animal experimental manipulations were conducted in accordance with the principles and procedures outlined with the guidelines for animal experiments of the EU directives and the permission from The administration of the Republic of Slovenia for food safety, veterinary and plant protection (permission No.: 34401-4/2012/2).

\section{In vivo electrochemotherapy protocol using noninvasive electroporation induced by PEMF or conventional electroporation}

Seven days after subcutaneously induction of B16F10 melanoma tumors $\left(40 \mathrm{~mm}^{3}\right)$ mice were randomly divided into the experimental groups as follows: intravenously injection of saline solution alone (Control) or combined with electroporation induced pulsed electromagnetic field (PEMF), intravenously injection of CDDP (CDDP) or combined with electroporation induced PEMF (PEMF + CDDP). Noninvasive electroporation was performed 3 minutes after intravenous injection of chemotherapeutic drugs by magnetic field pulse generator (TESLA Stym, Iskramedical, Slovenia) connected to an applicator consisted of round coil with 72 turns. The generator supplied the applicator with pulses of electric current that generated time-varying magnetic field around the coil, which in turn induced an electric field in the treated tissue (Figure 1).

In order to obtain precise application of electroporation mice were initially anesthetized with inhalation anaesthesia in the induction chamber with $2 \%(\mathrm{v} / \mathrm{v})$ of isoflurane (Isoflurane; Piramal Healthcare UK Limited, London, UK) and afterwards the mouse muzzle was placed under inhalation tube to remain anesthetized during experiment. The applicator for electroporation was positioned over the tumor so that the tumor was in the middle of the applicator (Figure 1A).

Based on the preliminary experiments, where four different sequences of bipolar pulses of electric current alone or in the combination with bleomycin were tested (Supplementary Table 1,
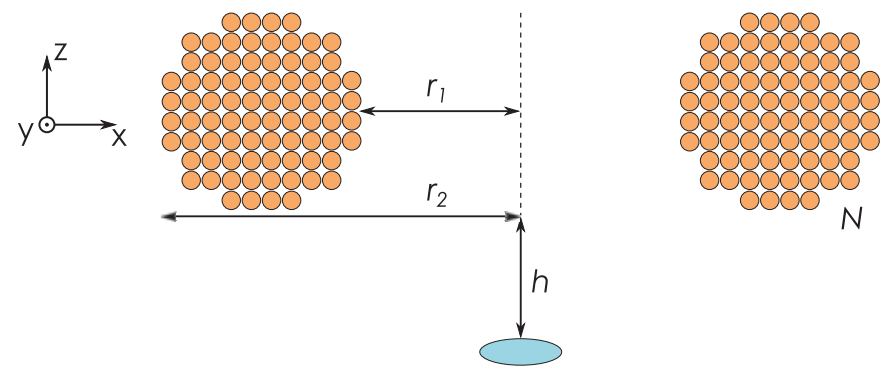

(A)
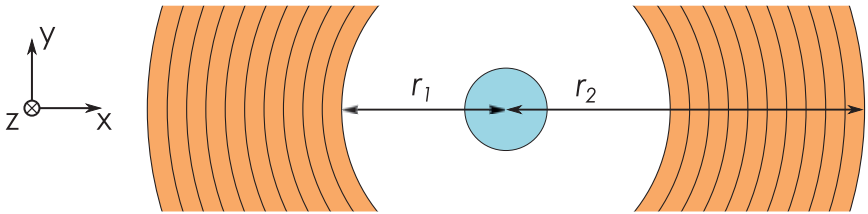

(B)

FIGURE 1. (A) Illustrated lateral view of multi-turn coil (colored orange) and the treated tumor (colored blue). Tumor was located in the center of the coil, i.e. $31 \mathrm{~mm}$ and $42 \mathrm{~mm}$ from the inner $\left(r_{1}\right)$ and outer boundary $\left(r_{2}\right)$ of the coil, respectively. The number of turns $(N)$ in coil was 72. Due to the casing of the applicator the coil was placed $23 \mathrm{~mm}$ above the tumor (h). (B) Illustrated view from above.

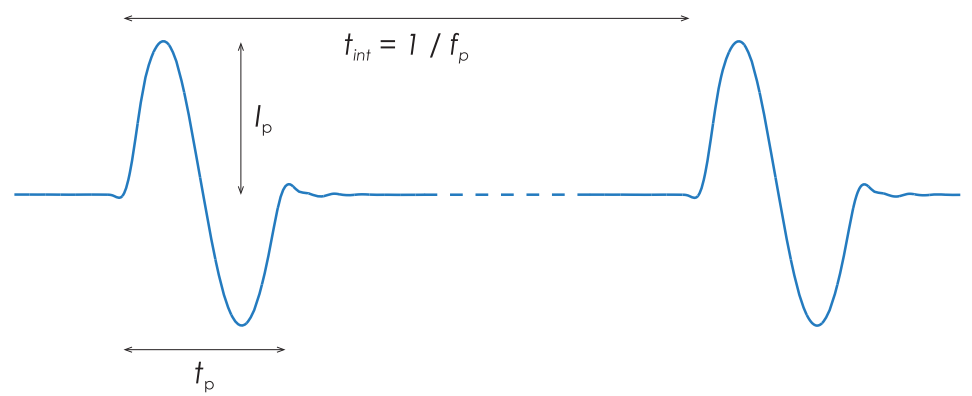

FIGURE 2. Sequence of bipolar electric pulses where $t_{p}$ is a duration of the pulse, $I_{p}$ is a pulse amplitude, $t_{\text {int }}$ is an interval between pulses and $t_{p}$ is a repetition frequency.

Supplementary Figure 1), the most promising sequence of bipolar pulses (Supplementary Figure 1) was used in the combination with CDDP. Briefly, the sequence had 480 bipolar pulses with duration of $t_{\mathrm{p}}=340 \mu \mathrm{s}$, with a peak of $I_{\mathrm{p}}=400 \mathrm{~A}$, repetition frequency $\left(f_{\mathrm{p}}\right)$ of $80 \mathrm{~Hz}$ and duration of each sequence $\left(t_{\mathrm{s}}\right)$ of $6 \mathrm{~s}$ (Figure 2). Electric pulses were measured using an oscilloscope (WavePro 7300A, LeCroy, Chestnut Ridge, NY) and current probe CWT Rogowski Current Transducer (Powertek, UK).

In previous experiments, groups of positive controls, such as conventional electroporation (EP) and the combination of EP with CDDP (ECT), were obtained. The growth of untreated tumors (tumor doubling time (the time in which tumor reaches twice of the initial volume, DT) in control was $1.4 \pm 0.2, \mathrm{n}=6$ ) and of CDDP treated tumors alone $(2.3 \pm 0.1, \mathrm{n}=4)$ in that independent experiment (data previously not published) were comparable 
to the experiment performed with PEMF treatment). In the conventional electrochemotherapy protocol three minutes after intravenously injection of CDDP eight square wave electric pulses at $1300 \mathrm{~V} / \mathrm{cm}$ voltage to distance ratio, $100 \mu$ s long and $1 \mathrm{~Hz}$ (Cliniporator ${ }^{\mathrm{TM}}$, IGEA s.r.l., Carpi, Italy) were applied by plate electrodes $(\mathrm{d}=8 \mathrm{~mm})$ to the tumors. Electric pulses were delivered in perpendicular orientation $(4+4)$ and good contact between the electrodes and tumor was assured using conductive gel.

\section{Determination of magnetic and electric field in the tumor}

Time-varying magnetic field and induced electric field of PEMF in the tumor was determined by means of numerical modelling. Numerical model of the applicator was modelled as multi-turn coil node which is a lumped model for tightly wound 72 wires separated by electrical insulator. Numerical model of the tumor was represented by an ellipsoid (Figure 1). Since volume of mice tumors varied from 30 to $40 \mathrm{~mm}^{3}$ an average volume, i.e. $35 \mathrm{~mm}^{3}$, was used in the numerical model of the tumor. Bipolar pulse (Figure 2) was used as electric current in the numerical model of the applicator. Calculations of time-varying magnetic field and induced electric field were performed using finite element method on a desktop PC (Windows 8.1, 3.50 GHz, 32 GB RAM) using commercial finite element software package COMSOL Multiphysics 5.1 (COMSOL AB, Stockholm, Sweden).

\section{Treatment evaluation}

The muscle contraction during PEMF treatment and conventional EP, tumor growth after therapy, skin area above the tumor and $2 \mathrm{~cm}$ in diameter around the tumor exposed to PEMF or conventional EP and the general well-being of animals (consumption of water and food, weight loss) were monitored during the experiment. Tumor growth was followed by measuring three mutually orthogonal tumor diameters $(a, b$, and c) with a Vernier caliper, every day. The tumor volumes were calculated by the formula:

$V=\frac{\prod \times a \times b \times c}{6}$.

The arithmetic mean of the tumor volumes and the standard error of the mean (SE) were calculated for each experimental group for each measurement day. The tumor growth delay was determined for each individual tumor by subtracting the average DT of the control group from the DT of each individual tumor.

\section{Platinum determination in the serum and tumors}

The measurements of platinum accumulation in the serum, tumors, platinum bound to the DNA in the cells and in extracellular fraction were performed by inductively coupled plasma mass spectrometry (ICP-MS, Agilent Technologies, model 7700x, Tokyo, Japan). ${ }^{195} \mathrm{Pt}$ isotope was monitored. At optimized instrumental parameters, instrumen-

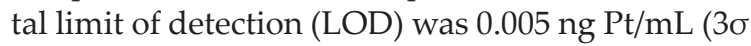
of the blanks). The linearity of the signal was confirmed from LOD to $10 \mu \mathrm{g} \mathrm{Pt} / \mathrm{mL}$. Repeatability of the measurements was better than $3 \%$.

The platinum uptake in tumors and its total concentration in the serum were measured 1 hour after the treatment of mice with intravenously injection of CDDP, electroporation induced by PEMF or the combination of those therapies (PEMF + CDDP). The blood was collected with glass capillary from intraorbital sinus (3-8 samples per group) and was coagulated at room temperature for two hours. Thereafter the blood was centrifuged at $3000 \mathrm{rpm}$ for 10 minutes and serum was collected and stored at the temperature $-20^{\circ} \mathrm{C}$. On the day of measurements total amount of serum samples were digested in $1 \mathrm{~mL}$ of $1: 1$ mixture of $65 \%$ nitric acid (MERCK KgaA, Dermstadt, Germany) and 30\% hydrogen peroxide (MERCK KgaA, Dermstadt, Germany) by incubation at $90^{\circ} \mathrm{C}$ for 48 hours. Obtained clear solutions were diluted with Milli-Q water before analysis.

For platinum determination in tumors, animals were sacrificed after the blood collection. The tumors (3-8 tumors per group) were excised and removed from the overlying skin. Each tumor was weighed, and placed into a $15 \mathrm{~mL}$ graduated polyethylene tube. For tumors digestion, the same procedure as for serum was applied, with the exception that $2 \mathrm{~mL}$ instead of $1 \mathrm{~mL}$ of $1: 1$ mixture of $65 \%$ nitric and $30 \%$ hydrogen peroxide was used. Before analysis samples were diluted with Milli-Q water.

\section{Determination of platinum bound to the DNA in the tumor cells and the extracellular fraction (fluid)}

The tumors were obtained as described in the chapter above, weighed and immediately mechan- 
ically disintegrated. The sample was washed with $3 \mathrm{~mL}$ of freshly prepared PBS and filtered through the cell strainer with pore size of $40 \mu \mathrm{m}$ (Corning Incorporated, Life Sciences, Durham, USA). The obtained cells in suspension were centrifuged at $1500 \mathrm{rpm}$ for 10 minutes. Collected cells were used for the fast DNA isolation by salting-out protocol. Briefly, cells were lysed with lysis buffer $(10 \mathrm{mM}$ Tris- $\mathrm{HCl}, 1 \mathrm{mM}$ ethylenediaminetetraacetic acid [EDTA], 1\% sodium dodecyl sulfate [SDS]) with proteinase $\mathrm{K}(20 \mu \mathrm{g})$ for 30 minutes at $55^{\circ} \mathrm{C}$ by constant shaking. After the samples were cooled down proteins were precipitated by adding of $120 \mu \mathrm{L} 4 \mathrm{M}$ $\mathrm{NaCl}$ and shaken for 15 seconds. Precipitated proteins were centrifuged at $13000 \mathrm{rpm}$ for 6 minutes. Supernatant was collected and centrifuged one times more at $13000 \mathrm{rpm}$ for 6 minutes. In addition, DNA was precipitated with $1 \mathrm{~mL}$ of ethanol (70\%) for 2 min by gentle mixing of tube and centrifuged at $13000 \mathrm{rpm}$ for 2 minutes. Precipitated DNA was washed with additional $1 \mathrm{~mL}$ of ethanol (70\%) and centrifuged at $13000 \mathrm{rpm}$ for 2 minutes. The pellet of DNA was dried out, resuspended in $100 \mu \mathrm{L}$ of distilled water, digested under the same procedure as serum and the concentration determined in diluted samples by ICP-MS.

The rest of two fractions, supernatant and the interstitial fraction on the top of the cell strainer, named as extracellular fraction, were collected and stored at $-20^{\circ} \mathrm{C}$ till the digestion with the mixture of nitric acid and hydrogen peroxide (see the section above).

\section{Statistical analysis}

All data were tested for normal distribution with the Shapiro-Wilk test. A t-test and one-way analysis of variance followed by a Holm-Sidak test were used for evaluation of the differences between the experimental groups. A p value less than 0.05 was considered significant. SigmaPlot Software (Systat Software, Chicago, IL, USA) was used for statistical analysis and graphical representation.

\section{Results}

\section{Antitumor effectives of electrochemotherapy mediated by PEMF}

Exposure of tumors to PEMF or conventional EP, performed 3 minutes after intravenous injection of CDDP, resulted in significant tumor growth delay, up to 3 days compared to untreated tumors, as well as compared to monotherapies. Nevertheless
TABLE 1 Tumor doubling times of melanoma B16F10 tumors after treatment with CDDP or combined with electroporation induced by PEMF.

\begin{tabular}{|c|c|c|c|c|}
\hline Group & $n$ & DT (Mean \pm SE) & GD & $P(<0.05)$ \\
\hline Control" & 12 & $1.5 \pm 0.1$ & & \\
\hline CDDP* $4 \mathrm{mg} / \mathrm{kg}$ & 12 & $2.2 \pm 0.2$ & 0.7 & \\
\hline PEMF & 9 & $1.9 \pm 0.1$ & 0.4 & \\
\hline PEMF + CDDP & 10 & $3.8 \pm 0.1$ & 2.3 & $\begin{array}{l}<0.001 \\
\text { (to PEMF) }\end{array}$ \\
\hline EP* & 12 & $2.2 \pm 0.3$ & 0.7 & \\
\hline ECT CDDP* & 8 & $4.5 \pm 0.2$ & 3.0 & $\begin{array}{c}<0.009 \\
\text { (to PEMF + CDDP) }\end{array}$ \\
\hline
\end{tabular}

CDDP = intravenously injection of cisplatin $(4 \mathrm{mg} / \mathrm{kg}) ; \mathrm{PEMF}=$ pulsed electromagnetic field treatment: PEMF + CDDP = PEMF after intravenously injection of CDDP. EP = electric pulses treatment; $E C T=$ electrochemotherapy, $E P$ after intravenously injection of $C D D P$; $D T=$ tumor doubling time; $G D=$ tumor growth delay; $p<0.05$ statistically significant difference; "Data pooled from separate experiments after checking that DT in control and CDDP treatment alone were comparable.

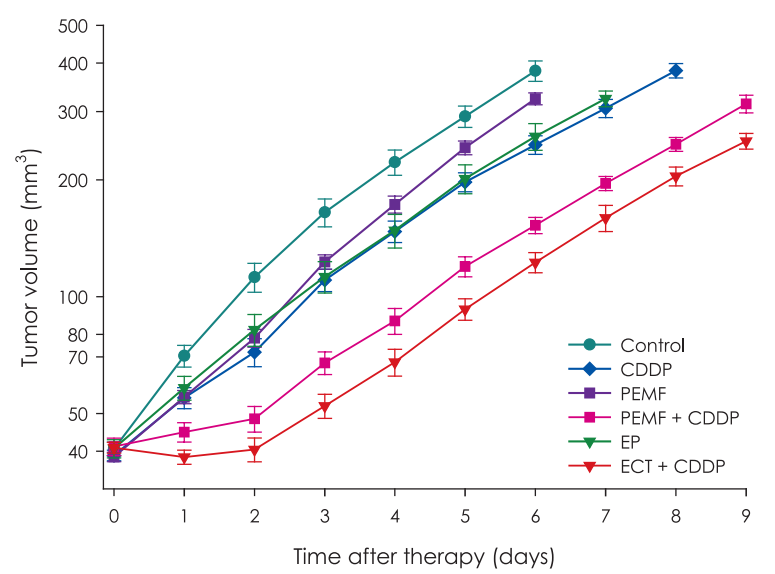

FIGURE 3. Antitumor effectiveness of electrochemotherapy with CDDP mediated by PEMF in mouse melanoma Bl6F10. Data were collected from two individual experiments and each point on graph represents mean and standard error of the mean $(A M \pm S E)$. Each group consisted at least of 8 animals.

CDDP = intravenously injection of cisplatin $(4 \mathrm{mg} / \mathrm{kg}) ; \mathrm{ECT}=$ electrochemotherapy, $\mathrm{EP}$ after intravenously injection of CDDP; $E P=$ electric pulses treatment; $P E M F=$ pulsed electromagnetic field treatment; PEMF + CDDP = PEMF after intravenously injection of CDDP

significantly higher antitumor effect was obtained after conventional electrochemotherapy compared to electrochemotherapy mediated by PEMF. Treatment of tumors with CDDP alone or exposure to PEMF or conventional EP had no significant effect on tumor growth (Table 1, Figure 3).

The application of PEMF did not exert muscle contraction, indicating on painless treatment procedure. Additionally, all treatments were well tolerated by animals, since no body weight loss or any detectable changes in skin, exposed to PEMF treatment during observation period was obtained, indicating that there was no systemic toxicity after treatments. 


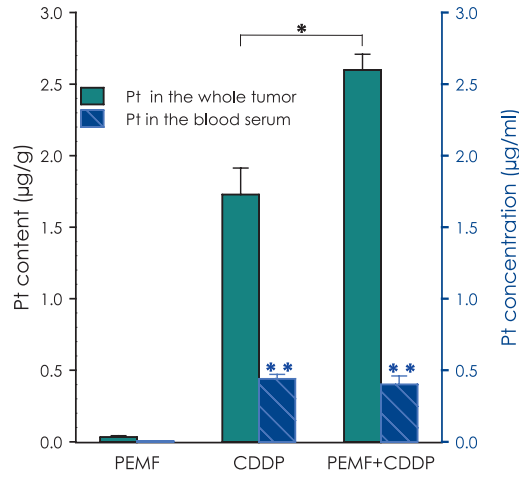

FIGURE 4. Platinum (Pt) accumulation in tumor and serum after electroporation induced by PEMF. Each group consisted from 3-8 animals. Data represent mean and standard error of the mean $(A M \pm S E)$.

CDDP = intravenously injection of cisplatin $(4 \mathrm{mg} /$ $\mathrm{kg}) ;$ PEMF = pulsed electromagnetic field treatment: PEMF + CDDP = PEMF after intravenously injection of CDDP. ${ }^{*}=p<0.05$ statistically significant difference; ${ }^{* *}=p<0.05$ statistically significant difference to measured Patinum (Pt) content in the whole tumor

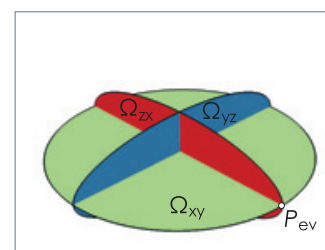

(A)

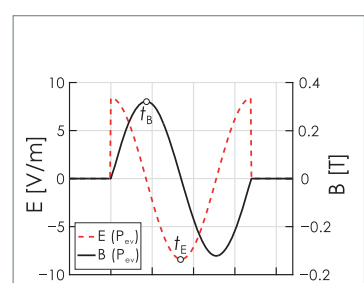

(C)

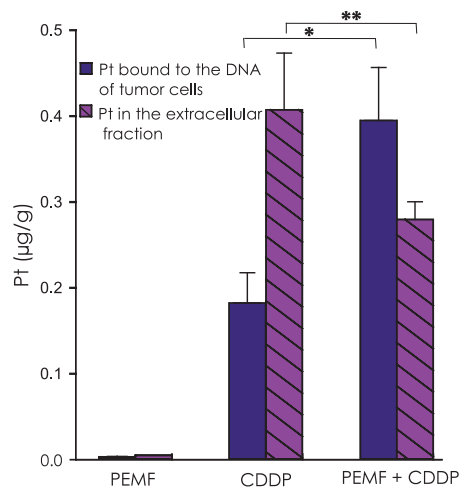

FIGURE 5. Platinum (Pt) bound to the DNA in tumor cells representing intracellular fraction and Pt content in extracellular fractions after electroporation induced by PEMF. Data represent mean and standard error of the mean $(A M \pm S E)$. Each group consisted from 3-8 animals.

CDDP = intravenously injection of cisplatin $(4 \mathrm{mg} / \mathrm{kg}) ; \mathrm{PEMF}=$ pulsed electromagnetic field treatment: PEMF + CDDP = PEMF after intravenously injection $=p<0.05$ statistically significant difference
Overall, the antitumor effect CDDP, i.e. the chemotherapeutic drug used, in the electrochemotherapy performed by PEMF was significantly increased in comparison to monotherapies, presumably due to facilitated transport of CDDP. However, electrochemotherapy after conventional EP was more effective than PEMF mediated electroporation.

\section{Determination of platinum in the serum and tumor after electroporation induced by PEMF}

In order to determine whether electroporation induced by PEMF facilitates drug delivery into the cells, as the underlying antitumor mechanism, $\mathrm{Pt}$ accumulation in the serum and tumors with plasma mass spectrometry was determined. First, $\mathrm{Pt}$ was measured as indicator of $\mathrm{CDDP}^{39}$ in whole tumors and plasma of the blood in mice. Intravenous CDDP injection demonstrated the drug accumulation in tumors, and electroporation induced by PEMF as successful method for increasing Pt accumulation in whole tumors, one hour after the drug administration (Figure 4). A statistically significant increase in platinum content in the tumors treated by electroporation induced by PEMF was observed, however this measurement does not indicate whether the electroporation induced by PEMF in fact facilitated drug delivery into the cells.

To prove that electroporation induced by PEMF facilitates transmembrane transport of CDDP, the extracellular and intracellular $\mathrm{Pt}$ amounts were measured. After mechanically disintegration of tumors, the suspension of tumor cells and extracellular fractions were obtained, and Pt was measured in both fractions. The concentration of the Pt detected in extracellular fraction statistically significantly decreased after electroporation induced by PEMF (Figure 5). Moreover, the Pt bound to DNA as indicator of the drug bound to the intracellular target was significantly increased after the electroporation induced by PEMF (Figure 5). Approximately 2 times higher values of Pt bound to DNA were obtained.

\section{Estimation of PEMF in the tumor}

Simulation results of PEMF in the tumor are presented in Figure 6. Electric field distribution in the tumor was linearly decreasing from the boundary of the tumor towards the center with a peak value of $8.6 \mathrm{~V} / \mathrm{m}$ on the tumor boundary. Magnetic flux density had a peak value of $0.3 \mathrm{~T}$ and its distribu-

FIGURE 6. (A) Evaluation surfaces $\left(\Omega_{\mathrm{xy}}, \Omega_{\mathrm{y} \mathrm{z}^{\prime}} \Omega_{\mathrm{zx}}\right.$ ) in three different planes (xy, yz, $\mathrm{zx}$ ) where electric field distribution and magnetic flux density were simulated by means of numerical modelling. (B) Distribution of induced electric field in evaluation surfaces when it reached its peak at $t_{E}$. (C) Time course of magnetic flux density and induced electric field at evaluation point $P_{\mathrm{ev}}$. Time points when magnetic flux density and induced electric field reached its maximum are marked with $t_{\mathrm{E}}$ and $t_{\mathrm{B}}$ respectively. (D) Distribution of magnetic flux density in evaluation surfaces when it reached its peak at $t_{B}$. 
tion in evaluation surfaces remained homogeneous through the whole surface of the tumor.

\section{Discussion}

This study demonstrated the use of contactless pulsed electromagnetic field (PEMF) treatment as an approach to achieve electroporation of melanoma tumor tissue, which increases drug uptake in vivo. We evaluated for the first time the antitumor effectiveness of electrochemotherapy obtained by PEMF after systemic injection of chemotherapeutic drug, CDDP, which is used in conventional electroporation protocol. Furthermore, we proved that the antitumor effect was related to increased drug uptake into the electroporated tumor cells, demonstrated by increased amount of $\mathrm{Pt}$ bound to the DNA. Thus PEMF treatment can be used (once optimized) for noninvasive drug delivery in vivo, which may be important for research where delicate tissues and organs needs to be avoided and for clinical applications, since it is noninvasive, contactless and painless compared to the classical electroporation using different electrodes.

Potential use of strong time-varying magnetic field which induced electric field to increase transmembrane molecular transport (i.e. electroporation), was already suggested before. ${ }^{30,34-36,38}$ In order to expand the use of contactless PEMF induced electroporation as drug delivery method the melanoma B16F10 tumor model in vivo was chosen, as it represents a great challenge in the treatment of human melanoma. ${ }^{1,14,40-43}$ We have shown that magnetic field generated by round coil which induced the 480 bipolar pulses, at frequency of $80 \mathrm{~Hz}$, pulse duration of $340 \mu \mathrm{s}$, significantly improved the antitumor effectiveness of electrochemotherapy with CDDP. Our results are in accordance with in vitro study, where much stronger PEMF (6.1 T) was indicated as delivery method for therapeutic molecules in human pathogenic fungi. Namely, the synergistic effect of simultaneos treatment of 200 applied magnetic field pulses at frequency of $35 \mathrm{~Hz}$ and drug was observed. ${ }^{36}$ However, the application of stronger magnetic field (up to $16.4 \mathrm{~T}$ ) did not result in better membrane permeabilization. ${ }^{35}$ The membrane permeabilization seems to be dependent more on the shape, number and frequency of generated pulses ${ }^{34-36}$ in addition to the amplitude of induced electric field, similar to the conventionally generated bipolar pulses. ${ }^{44}$ In addition, bipolar pulses were demonstrated two times more effective as monopolar and almost equally effective as conventional square wave pulses. ${ }^{37}$ In fact, for the almost equal membrane permeabilization larger number of short duration induced bipolar electric pulses at higher frequency has to be delivered ${ }^{37}$ Similarly, in our study the obtained antitumor effectiveness of cisplatin by using short and larger number of induced electric pulses at higher frequency in comparison to conventional electric pulses was comparable. Furthermore, it is known that simple round coils induce less focused and lower peak electric field than figure-of-eight coils. ${ }^{45,46}$ It was also demonstrated in vitro that by using figure-of-eight coils the increased transmembrane molecular transport could be obtained by pulses of lower frequencies and larger number. ${ }^{34}$ However, we and others ${ }^{34}$ have shown that by increasing the number of pulses at the same frequency the effect of electroporation can be improved (supplementary data).

Presently, in conventional electrochemotherapy protocols mainly square wave or monotonically decreasing electric pulses are delivered through plate or needle electrodes to the cells or tissues. ${ }^{15,18,23,25} \mathrm{On}$ the contrary, only a few studies were performed with bipolar electric pulses for the electroporation of cells in vitro and tissues in vivo. ${ }^{44,47-55}$ In general, the lower pulse amplitudes were needed for effective electroporation of cells in vitro with respect to unipolar pulses. ${ }^{48,51}$ Besides that, the pulse shape played important role in electroporation of cells as well. ${ }^{34,51,56}$ It was shown that electroporation, cell death and the uptake of Lucifer Yellow occurred by using the rectangular bipolar pulses at lowest, the sine bipolar pulses at medium and the triangular bipolar pulses at the highest pulses amplitudes. ${ }^{44}$ Bipolar pulses were already applied successfully in electrochemotherapy for human and veterinary clinic. ${ }^{49,57,58}$

In fact, the combination of PEMF induced electroporation with CDDP had significant antitumor effectiveness, whereas the application of PEMF or the drug alone had none. The antitumor effectiveness of electrochemotherapy of applied PEMF (480 bipolar pulses, at frequency of $80 \mathrm{~Hz}$, pulse duration of $340 \mu \mathrm{s}$ ) was presumably due to improved membrane permeabilization of cells in the tissue, since the monotherapies alone had very little but no significant effect on tumor growth in comparison to control. Similarly, preclinical and clinical studies performed in conventional scheme of electrochemotherapy with CDDP have shown great antitumor effectiveness of electrochemotherapy on different tumor types ${ }^{15,42,59-62}$, mostly due to direct cytotoxic effect on tumor cells. ${ }^{60-62}$ It has been demonstrated 
that after conventional electroporation the cytotoxicity of CDDP could be improved by 70-times. ${ }^{61-63}$ Nevertheless, in our study sufficient antitumor effectiveness on melanoma B16F10 was obtained with electrochemotherapy after PEMF induced electroporation, despite the effect was significantly lower compared to that obtained after conventional electrochemotherapy with CDDP. However, calculations suggest that levels of electric field are 4 orders of magnitude lower than those associated to classical electroporation ${ }^{64}$, but tend to be high enough to induce electroporation. On the other side, while using electric field bellow $0.09 \mathrm{~V} / \mathrm{cm}$ at the position of tumor site, we suspect there was no possible occurrence of irreversible electroporation or thermal effect, as obtained at much higher electric field induced by PEMF (up to $40 \mathrm{~V} / \mathrm{cm}$ ). ${ }^{38}$ Even more, we speculate that PEMF could be improved by positioning of tumor towards the edge of the coil, where based on calculations the highest strength of magnetic field could be obtained, which consequently could induce higher electric field strength and thus, even more cells could be successfully electroporated at deeper parts of tumor tissue. Therefore, further studies to optimize the PEMF are warranted.

Even though it was previously known that the time varying magnetic field could induce electroporation $^{34-37,65}$, affect the cytoskeleton and intracellular signal transduction $28,32,66,67$, the mechanisms of its action in the combination with CDDP have not been studied yet. Therefore, to clarify the antitumor effectiveness of electrochemotherapy the measurements of platinum amount after electroporation induced by PEMF were performed. Observed significant 1.6-fold increased platinum uptake into melanoma B16F10 tumors after electroporation induced by PEMF indirectly confirmed membrane permeabilization of the tumor cells and thus, its correlation with the antitumor effectiveness of electrochemotherapy. Our results are in accordance with results reported in other studies, where up to two times higher platinum uptake was obtained in sarcoma SA-1 and fibrosarcoma LPB tumors after conventional electrochemotherapy with CDDP..$^{60,61}$ Even though lower increase of platinum amount in tumors was obtained after PEMF in comparison to conventional electrochemotherapy ${ }^{60,61}$, the final amount of platinum in the tumors was comparable. The difference of platinum amount in tumors treated only with CDDP, might be tumor type dependent, since melanoma tumors are well vascularized and contained large spherical cells, with less surrounding extracellular matrix component in com- parison to stiff SA-1 and LPB tumors with small spindle-shaped cells and high content of extracellular matrix component. ${ }^{68,69}$ On the other hand, the amount of platinum in the serum was significantly up to 6-times lower compared to tumor tissue. Therefore we could assume that excess of the drug which was not entrapped in the tumors after electroporation was washed out with similar kinetic as in nonelectroporated tumors. Moreover, our results indicated that CDDP in the cells reached its main intracellular target DNA, in fact significantly two times higher amount of $\mathrm{Pt}$ was bounded to the DNA in PEMF and CDDP treated tumors than in CDDP only treated tumors. At the same time, as expected the pool of Pt amount in the extracellular fraction of these tumors was lowered, up to 1.4- times. Thus, the increased Pt uptake in the cells and its binding to the DNA could indicate the main reason for antitumor effectiveness of electrochemotherapy mediated by PEMF.

Presently, it is not clear if the membrane permeabilization obtained after application of time varying magnetic field occurs only due to induced electric field as in conventional electroporation or due to direct effect of magnetic field with the plasma membrane and surrounding ions. Thus, the precise mechanism of cisplatin uptake after electroporation mediated by PEMF into the cells remains unclear. Obtained Pt amount in the tumor cells after treatment with CDDP only could be ascribed to passive diffusion and active transport mechanisms of cisplatin through the membrane, which are carrier-mediated through formed pores or via endocytosis..$^{70,71}$ In addition, it has been demonstrated that exposure of cells to train of unipolar pulsed low electric fields at strength from 1.2 up to $20 \mathrm{~V} / \mathrm{cm}$ can induce electro-endocytosis. ${ }^{72,73}$ Thus, we suspect that generated bipolar electric field of just below $0.09 \mathrm{~V} / \mathrm{cm}$ by PEMF might also trigger endocytosis besides membrane permeabilization which enables the internalization of cisplatin in the cell and contributes partially to the increase of platinum amount.

In conclusion, our results show that PEMF at magnetic field below of $1 \mathrm{~T}$ was sufficient to achieve membrane permeabilization of tumor cells, thus, small molecules such as drug (CDDP) improved delivery and cellular uptake in solid tumors was enabled. Due to simple, contactless, painless, focused local application of PEMF, better field distribution irrespective of tissue type and thus, achieving electric field strength for membrane permeabilization can be established in deeper parts of tissue. However this approach has the limitation that the strength of the magnetic field decreases rapidly 
with distance from the coil which has to be taken into account by designing coils ${ }^{74}$ in order to achieve successful permeabilization at a greater tissue depth. PEMF might thus represent an alternative to conventional electroporation with electric fields in electrochemotherapy. However further studies are needed to improve the equipment, to optimize and establish precise protocols of drug application and PEMF parameters, as well as to reveal the effects of PEMF on variety of normal and tumor tissues.

\section{Acknowledgements}

The work was performed in the scope of The European Associated Laboratory entitled Pulsed Electric Fields Applications in Biology and Medicine (LEA-EBAM). This research was supported by the Slovenian Research Agency under program grants (P2-0249 and P3-0003) and by Slovenian Ministry of Education, Science and Sport under program grant M-1330E. The authors acknowledge Tanja Dolinšek and Lara Prosen for technical help in preparation of tumor samples subjected to platinum measurements. The research has been achieved due to the networking efforts of the COST TD1104 Action (www.electroporation.net). The paper was presented at the $1^{\text {st }}$ World Congress on Electroporation and Pulsed Electric Fields in Biology, Medicine, and Food \& Environmental Technologies, September 6 to 10, 2015, Portorož, Slovenia (wc2015.electroporation.net) organized by COST TD1104 Action (www.electroporation. net), supported by COST (European Cooperation in Science and Technology).

\section{References}

1. Yarmush ML, Golberg A, Sersa G, Kotnik T, Miklavcic D. Electroporationbased technologies for medicine: principles, applications, and challenges. Ann Rev Biomed Eng 2014; 16: 295-320.

2. Neumann E, Kakorin S, Toensing K. Fundamentals of electroporative delivery of drugs and genes. Bioelectrochem Bioenerg 1999; 48: 3-16.

3. Weaver JC. Electroporation of cells and tissues. IEEE Trans Plasma Sci 2000; 28: 24-33.

4. Kotnik T, Kramar P, Pucihar G, Miklavcic D, Tarek M. Cell membrane electroporation-part 1: the phenomenon. IEEE Electr Insul M 2012; 28: 14-23.

5. Delemotte L, Tarek M. Molecular dynamics simulations of lipid membrane electroporation. J Membr Biol 2012; 245: 531-43.

6. Haberl JS, Abbas M. Development of graphical indices for viewing building energy data: Part II. J Sol Energy Eng Trans ASME 1998; 120: 162-7.

7. Miklavcic D, Mali B, Kos B, Heller R, Sersa G. Electrochemotherapy: from the drawing board into medical practice. Biomed Eng Online 2014; 13: 29.

8. Scheffer HJ, Nielsen K, de Jong MC, van Tilborg AA, Vieveen JM, Bouwman AR, et al. Irreversible electroporation for nonthermal tumor ablation in the clinical setting: a systematic review of safety and efficacy. J Vasc Interv Radiol 2014; 25: 997-1011; quiz 1011.
9. Beebe SJ, Fox PM, Rec $\amalg$, Willis EL, Schoenbach KH. Nanosecond, highintensity pulsed electric fields induce apoptosis in human cells. FASEB $J$ 2003; 17: 1493-5.

10. Zupanic A, Kos B, Miklavcic D. Treatment planning of electroporation-based medical interventions: electrochemotherapy, gene electrotransfer and irreversible electroporation. Phys Med Biol 2012; 57: 5425-40.

11. Kos B, Voigt $P$, Miklavcic D, Moche M. Careful treatment planning enable safe ablation of liver tumors adjacent to major blood vessels by percutaneous irreversible electroporation (IRE). Radiol Oncol 2015; 49: 234-41.

12. Miklavcic D. Network for development of electroporation-based technologies and treatments: COST TD1104. J Membr Biol 2012; 245: 591-8.

13. Curatolo P, Quaglino P, Marenco F, Mancini M, Nardo T, Mortera C, et al. Electrochemotherapy in the treatment of Kaposi sarcoma sutaneous lesions: a two-center prospective phase II trial. Ann Surg Oncol 2012; 19: 192-8.

14. Marty M, Sersa G, Garbay JR, Gehl J, Collins CG, Snoj M, et al. Electrochemotherapy - an easy, highly effective and safe treatment of cutaneous and subcutaneous metastases: results of ESOPE (European Standard Operating Procedures of Electrochemotherapy) study. EJC Suppl 2006; 4: 3-13.

15. Sersa G, Miklavcic D, Cemazar M, Rudolf Z, Pucihar G, Snoj M. Electrochemotherapy in treatment of tumours. Eur J Surg Oncol 2008; 34: 232-40.

16. Gehl J, Geertsen PF. Palliation of haemorrhaging and ulcerated cutaneous tumours using electrochemotherapy. EJC Supp/ 2006; 4: 35-7.

17. Valpione S, Campana LG, Pigozzo J, Chiarion-Sileni V. Consolidation electrochemotherapy with bleomycin in metastatic melanoma during treatment with dabrafenib. Radiol Oncol 2015; 49: 71-4.

18. Mir LM, Gehl J, Sersa G, Collins CG, Garbay JR, Billard V, et al. Standard operating procedures of the electrochemotherapy: Instructions for the use of bleomycin or cisplatin administered either systemically or locally and electric pulses delivered by the Cliniporator (TM) by means of invasive or non-invasive electrodes. EJC Suppl 2006; 4: 14-25.

19. Edhemovic I, Brecelj E, Gasljevic G, Music MM, Gorjup V, Mali B, et al. Intraoperative Electrochemotherapy of colorectal liver metastases. J Surg Oncol 2014; 110: 320-7.

20. Mali B, Jarm T, Snoj M, Sersa G, Miklavcic D. Antitumor effectiveness of electrochemotherapy: a systematic review and meta-analysis. Eur J Surg Oncol 2013; 39: 4-16.

21. Miklavcic D, Snoj M, Zupanic A, Kos B, Cemazar M, Kropivnik M, et al. Towards treatment planning and treatment of deep-seated solid tumors by electrochemotherapy. Biomed Eng Online 2010; 9.

22. Miklavcic D, Sersa G, Brecelj E, Gehl J, Soden D, Bianchi G, et al Electrochemotherapy: technological advancements for efficient electroporation-based treatment of internal tumors. Med Biol Eng Comput 2012; 50: $1213-25$.

23. Cemazar M, Tamzali Y, Sersa G, Tozon N, Mir LM, Miklavcic D, et al. Electrochemotherapy in veterinary oncology. J Vet Intern Med 2008; 22: 826-31.

24. Tamzali Y, Borde L, Rols MP, Golzio M, Lyazrhi F, Teissie J. Successful treatment of equine sarcoids with cisplatin electrochemotherapy: A retrospective study of 48 cases. Equine Vet J 2012; 44: 214-20.

25. Tozon N, Kodre V, Sersa G, Cemazar M. Effective treatment of perianal tumors in dogs with electrochemotherapy. Anticancer Res 2005; 25: 839-45.

26. Tozon N, Pavlin D, Sersa G, Dolinsek T, Cemazar M. Electrochemotherapy with intravenous bleomycin injection: an observational study in superficial squamous cell carcinoma in cats. J Feline Med Surg 2014; 16: 291-9.

27. Rebersek M, Miklavcic D, Bertacchini C, Sack M. Cell membrane electroporation-part 3: the equipment. IEEE Electr Insul M 2014; 30: 8-18.

28. Belton M, Prato FS, Rozanski C, Carson JJL. Effect of $100 \mathrm{mT}$ homogeneous static magnetic field on $\left[\mathrm{Ca}^{2+}\right](\mathrm{c})$ response to ATP in HL-60 cells following GSH depletion. Bioelectromagnetics 2009; 30: 322-9.

29. Bodega G, Forcada I, Suarez I, Fernandez B. Acute and chronic effects of exposure to a 1-mT magnetic field on the cytoskeleton, stress proteins, and proliferation of astroglial cells in culture. Environ Res 2005; 98: 355-62.

30. Chen C, Evans JA, Robinson MP, Smye SW, O'Toole P. Electroporation of cells using EM induction of ac fields by a magnetic stimulator. Phys Med Biol 2010; 55: 1219-29. 
31. Dini L, Dwikat M, Panzarini E, Vergallo C, Tenuzzo B. Morphofunctional study of 12-O-tetradecanoyl-13-phorbol scetate (TPA)-induced differentiation of U937 cells under exposure to a $6 \mathrm{mT}$ static magnetic field. Bioelectromagnetics 2009; 30: 352-64.

32. Flipo D, Fournier M, Benquet C, Roux P, Le Boulaire C, Pinsky C, et al. Increased apoptosis, changes in intracellular $\mathrm{Ca}^{2+}$, and functional alterations in lymphocytes and macrophages after in vitro exposure to static magnetic field. J Toxicol Environ Health A 1998; 54: 63-76.

33. Ikehara T, Nishisako $H$, Minami $Y$, Ichinose $H$, Shiraishi T, Kitamura $M$, et al. Effects of exposure to a time-varying 1.5 T magnetic field on the neurotransmitter-activated increase in intracellular $\mathrm{Ca}^{2+}$ in relation to actin fiber and mitochondrial functions in bovine adrenal chromaffin cells. Biochim Biophys Acta 2010; 1800: 1221-30.

34. Towhidi L, Firoozabadi SMP, Mozdarani H, Miklavcic D. Lucifer Yellow uptake by $\mathrm{CHO}$ cells exposed to magnetic and electric pulses. Radiol Oncol 2012; 46: 119-25.

35. Novickij V, Grainys A, Kucinskaie-Kodze I, Zvirbliene A, Novickij J. Magnetopermeabilization of viable cell membrane using high pulsed magnetic field. IEEE Trans Magn 2015; 51(9).

36. Novickij V, Grainys A, Svediene J, Markovskaja S, Paskevicius A, Novickij J. Microsecond pulsed magnetic field improves efficacy of antifungal agents on pathogenic microorganisms. Bioelectromagnetics 2014; 35: 347-53.

37. Kardos TJ, Rabussay DP. Contactless magneto-permeabilization for intracellular plasmid DNA delivery in vivo. Human Vaccin Immunother 2012; 8 1707-13.

38. Novickij V, Grainys A, Novickij J, Markovskaja S. Irreversible magnetoporation of micro-organisms in high pulsed magnetic fields. IET Nanobiotechnol 2014; 8: 157-62.

39. Milacic R, Cemazar M, Sersa G. Determination of platinum in tumour tissues after cisplatin therapy by electrothermal atomic absorption spectrometry. $J$ Pharm Biomed Anal 1997; 16: 343-8.

40. Campana LG, Testori A, Mozzillo N, Rossi CR. Treatment of metastatic melanoma with electrochemotherapy. J Surg Oncol 2014; 109: 301-7.

41. Savoia P, Fava P, Nardo T, Osella-Abate S, Quaglino P, Bernengo MG. Skin metastases of malignant melanoma: a clinical and prognostic survey. Melanoma Res 2009; 19: 321-6.

42. Sersa G, Stabuc B, Cemazar M, Miklavcic D, Rudolf Z. Electrochemotherapy with cisplatin: clinical experience in malignant melanoma patients. Clin Cancer Res 2000; 6: 863-7.

43. Spratt DE, Spratt EAG, Wu SH, DeRosa A, Lee NY, Lacouture ME, et al. Efficacy of skin-directed therapy for cutaneous metastases from advanced cancer: a meta-analysis. J Clin Oncol 2014; 32: 3144-55.

44. Kotnik T, Pucihar G, Rebersek M, Miklavcic D, Mir LM. Role of pulse shape in cell membrane electropermeabilization. Biochim Biophys Acta 2003, 1614: $193-200$.

45. Jalinous R. Technical and Practical Aspects of Magnetic Nerve-Stimulation. $J$ Clin Neurophysiol 1991; 8: 10-25.

46. Ravazzani P, Ruohonen J, Grandori F, Tognola G. Magnetic stimulation of the nervous system: Induced electric field in unbounded, semi-infinite, spherical, and cylindrical media. Ann Biomed Eng 1996; 24: 606-16.

47. Arena CB, Sano MB, Rylander MN, Davalos RV. Theoretical considerations of tissue electroporation with high-frequency bipolar pulses. IEEE Trans Biomed Eng 2011; 58: 1474-82.

48. Chen C, Evans JA, Robinson MP, Smye SW, O'Toole P. Measurement of the efficiency of cell membrane electroporation using pulsed ac fields. Phys Med Biol 2008; 53: 4747-57.

49. Daskalov I, Mudrov N, Peycheva E. Exploring new instrumentation parameters for electrochemotherapy - attacking tumors with bursts of biphasic pulses instead of single pulses. IEEE Eng Med Biol Mag 1999; 18: 62-6.

50. Kotnik T, Miklavcic D, Mir LM. Cell membrane electropermeabilization by symmetrical bipolar rectangular pulses - part II. Reduced electrolytic contamination. Bioelectrochemistry 2001; 54: 91-5.

51. Kotnik T, Mir LM, Flisar K, Puc M, Miklavcic D. Cell membrane electropermeabilization by symmetrical bipolar rectangular pulses - part I. Increased efficiency of permeabilization. Bioelectrochemistry 2001; 54: 83-90.
52. Kuriyama S, Tsujinoue $H$, Toyokawa $Y$, Mitoro A, Nakatani T, Yoshiji $H$, et al. A potential approach for electrochemotherapy against colorectal carcinoma using a clinically available alternating current system with bipolar snare in a mouse model. Scand J Gastroenterol 2001; 36: 297-302.

53. Mathiesen I. Electropermeabilization of skeletal muscle enhances gene transfer in vivo. Gene Ther 1999; 6: 508-14.

54. Rizzuto G, Cappelletti M, Maione D, Savino R, Lazzaro D, Costa P, et al Efficient and regulated erythropoietin production by naked DNA injection and muscle electroporation. Proc Natl Acad Sci U S A 1999; 96: 6417-22.

55. Todorovic V, Kamensek U, Sersa G, Cemazar M. Changing electrode orientation, but not pulse polarity, increases the efficacy of gene electrotransfer to tumors in vivo. Bioelectrochemistry 2014; 100: 119-27.

56. Miklavcic D, Mir LM, Vernier PT. Electroporation-based technologies and treatments. J Membr Biol 2010; 236: 1-2.

57. Lanza A, Baldi A, Spugnini EP. Surgery and electrochemotherapy for the treatment of cutaneous squamous cell carcinoma in a yellow-bellied slider (Trachemys scripta scripta). J Am Vet Med Assoc 2015; 246: 455-7.

58. Spugnini EP, Citro G, Baldi A. Adjuvant electrochemotherapy in veterinary patients: a model for the planning of future therapies in humans. J Exp Clin Cancer Res 2009; 28:114.

59. Cemazar M, Golzio M, Escoffre JM, Couderc B, Sersa G, Teisse J. In vivo imaging of tumor growth after electrochemotherapy with cisplatin. Biochem Biophys Res Commun 2006; 348: 997-1002.

60. Cemazar M, Miklavcic D, Scancar J, Dolzan V, Golouh R, Sersa G. Increased platinum accumulation in SA-1 tumour cells after in vivo electrochemotherapy with cisplatin. Br J Cancer 1999; 79: 1386-91.

61. Kranjc S, Cemazar M, Grosel A, Scancar J, Sersa G. Electroporation of LPB sarcoma cells in vitro and tumors in vivo increases the radiosensitizing effect of cisplatin. Anticancer Res 2003; 23: 275-81.

62. Sersa G, Cemazar M, Miklavcic D. Antitumor effectiveness of electrochemotherapy with cis-diamminedichloroplatinum(II) in mice. Cancer Res 1995; 55: $3450-5$

63. Kranjc S, Cemazar M, Grosel A, Pipan Z, Sersa G. Effect of electroporation on radiosensitization with cisplatin in two cell lines with different chemo- and radiosensitivity. Radiol Oncol 2003; 37: 101-7.

64. Haberl S, Miklavcic D, Sersa G, Frey W, Rubinsky B. Cell membrane electroporation - Part 2: the applications. IEEE Electr Insul M 2013; 29: 29-37.

65. Novickij V, Grainys A, Novickij J, Lucinskis A, Zapolskis P. Compact microsecond pulsed magnetic field generator for application in bioelectronics. Elektronika IR Elektrotechnika 2013; 19: 25-8.

66. Morgado-Valle C, Verdugo-Diaz L, Garcia DE, Morales-Orozco C, DruckerColin $\mathrm{R}$. The role of voltage-gated $\mathrm{Ca}^{2+}$ channels in neurite growth of cultured chromaffin cells induced by extremely low frequency (ELF) magnetic field stimulation. Cell Tissue Res 1998; 291: 217-30.

67. Rotem A, Moses E. Magnetic stimulation of one-dimensional neuronal cultures. Biophys J 2008; 94: 5065-78.

68. Cemazar M, Golzio M, Sersa G, Escoffre JM, Coer A, Vidic S, et al Hyaluronidase and collagenase increase the transfection efficiency of gene electrotransfer in various murine tumors. Hum Gene Ther 2012; 23: 128-37.

69. Mesojednik S, Pavlin D, Sersa G, Coer A, Kranjc S, Grosel A, et al. The effect of the histological properties of tumors on transfection efficiency of electrically assisted gene delivery to solid tumors in mice. Gene Ther 2007; 14: 1261-9.

70. Abada P, Howell SB. Regulation of cisplatin cytotoxicity by Cu influx transporters. Met Based Drugs 2010; 2010: 317581.

71. Arnesano F, Losacco M, Natile G. An updated view of cisplatin transport. Eur J Inorg Chem 2013: 2701-11.

72. Antov $\mathrm{Y}$, Barbul A, Korenstein R. Electroendocytosis: stimulation of adsorptive and fluid-phase uptake by pulsed low electric fields. Exp Cell Res 2004; 297: 348-62.

73. Antov Y, Barbul A, Mantsur H, Korenstein R. Electroendocytosis: exposure of cells to pulsed low electric fields enhances adsorption and uptake of macromolecules. Biophys J 2005; 88: 2206-23.

74. Deng Z, De Lisanby SH, Peterchev AV. Electric field depth-focality trade off in transcranial magnetic stimulation: Simulation comparison of 50 coil designs. Brain Stimul 2013; 6: 1-13. 